\title{
Tailoring spatially unpolarized light on the Poincaré sphere: From equator states via meridian states to generalized great-circle states
}

\author{
Florian Kroh \\ Institute of Applied Physics, Technische Universität Darmstadt, 64289 Darmstadt, Germany \\ Markus Rosskopf \\ Institute of Applied Physics, Technische Universität Darmstadt, 64289 Darmstadt, Germany \\ Wolfgang Elsäßer (1) \\ Institute of Applied Physics, Technische Universität Darmstadt, 64289 Darmstadt, Germany \\ and School of Physics, Trinity College Dublin, Dublin 2, Ireland
}

(Received 31 July 2021; accepted 3 November 2021; published 22 November 2021)

\begin{abstract}
In this paper we investigate the generation, manipulation and invariance properties of spatially unpolarized light in the context of classical optics. We generate spatially randomly polarized light by exploiting the optical activity of a so-called Cornu depolarizer and demonstrate tailored, spatial polarization distributions on the Poincaré sphere. We begin with the generation of equator states of spatially unpolarized light, i.e., the manifold of the superposition of all linearly polarized light states in the spatial domain. A quarter-wave plate is utilized to transform these equator polarization states into meridian states and finally a second, subsequent quarter-wave plate results in tilted meridian states or generalized great-circle states on the Poincaré sphere. These results of all realized unpolarized states are visually confirmed by tomographic reconstruction of the spatially distributed polarization states on the Poincare sphere by extracting the data from the spatially resolved Stokes parameter measurements. Furthermore, all these experimental results are in excellent agreement with classical Mueller formalism calculations based on the Mueller matrices for the Cornu depolarizer and the wave plates, yielding the tailored manifold of polarization states on the Poincaré sphere's surface, confirming the naming of the unpolarized light states in accordance with geodesy. Therefore, we have realized a scheme for the tailored generation of spatially depolarized light based on the Cornu depolarizer and quarter-wave plates with interesting application perspectives and giving further insight into polarization.
\end{abstract}

DOI: 10.1103/PhysRevResearch.3.043131

\section{INTRODUCTION}

Polarization is even 170 years after Stokes still a fascinating hot topic at the center of investigations of the characteristics of electromagnetic waves and their applications. Recently, novel concepts in the field of polarization have been realized, such as full Poincaré beams [1], angular momentum states [2], and vector beams [3,4] and have stimulated developments in related fields such as plasmonics. Polarization is an essential degree of freedom in the generation of structured light [5], which itself has established its own field of research with a plethora of potential applications and the promise of tailoring light right at the source [6]. Furthermore, polarization can be regarded both under its pure, simple classical aspects, as well as nowadays even more under

\footnotetext{
*Corresponding author: elsaesser@physik.tu-darmstadt.de

Published by the American Physical Society under the terms of the Creative Commons Attribution 4.0 International license. Further distribution of this work must maintain attribution to the author(s) and the published article's title, journal citation, and DOI.
}

its quantum aspects [7]. Here, exactly at this border, thus bridging the quantum and classical nature of polarization, our research can be seen as leading to further insights as well as establishing perspectives for relevant applications.

Polarized light has been studied by analyzing higher order correlations [8,9] using theoretical concepts such as the Simon-Mukunda polarization gadget [10-14]. Besides the well-defined class of polarization states in space and time, socalled unpolarized or randomly polarized light has attracted attention, with respect to both fundamental aspects and applications [7]. Unpolarized light is generated in various ways. In the spatial domain, depolarizers are used to produce light that is fully polarized locally, but appears unpolarized on spatial average. In the temporal domain it is generated by means of rotating polarization optics, for instance [15]. Sunlight is an example of unpolarized light in nature.

While unpolarized light and depolarized light has a long history dating back to the 1930s [16-18], it has been in the focus of recent developments of applications such as the generation of vector beams $[3,4]$, in metrology $[19,20]$, and tomography of polarization states $[21,22]$. Unpolarized light has also very recently played a central role in Floquet engineering $[23,24]$ and in the shape of nonuniformly totally polarized 
beams (or full Poincaré beams) [25,26]. An extensive current survey of unpolarized light is given by Luis [27].

Recently, a new framework for the understanding of unpolarized light was developed by Shevchenko et al. [28,29], who experimentally demonstrated that for unpolarized light an instantaneous polarization state, represented by a single Stokes vector, exists and can be measured on the femtosecond scale. Based on this model, in the field of ghost imaging the theory of polarization correlations [30] was consolidated and used to implement applications such as ghost polarization communication [31].

From a theoretical point of view, unpolarized light has been formalized [32] and classified by Paul et al. [33-35] in two categories, depending on three invariance and symmetry properties which are accessible through Stokes parameter correlations [36,37]. Type II unpolarized light satisfies invariance with respect to the direction of propagation and handedness, while type I unpolarized (or "natural light," e.g., sunlight) additionally satisfies invariance with respect to phase changes. In the picture of the Poincaré sphere [38], type I unpolarized light relates to a spatial distribution uniformly covering the entire sphere, while type II unpolarized light displays other distributions, e.g., in the form of a ring encompassing the sphere. In this context, light scattered by compressed powders has been used to gain a better understanding of the correlations of spatially depolarized light [39]. Discriminating randomly polarized fields that underpin unpolarized light is possible by introducing measures such as the complex degree of mutual polarization [40]. Recently, we investigated temporally unpolarized light from a fiber amplifier and have successfully proven that it represents type I unpolarized light [41] according to these required invariance properties. Very recently, we also demonstrated the generation of type II spatially unpolarized light [42] by utilizing a Cornu depolarizer [43].

The polarization state of polarized light or spatially unpolarized light can be both represented on the Poincaré sphere [44], as either a single stationary vector or a polarization distribution on the sphere's surface, respectively. This representation is also used to model dynamic changes of the polarization state of monochromatic sources [45] as well as random light sources [46]. Here we use this Poincaré sphere depiction to discuss and realize experimentally unpolarized light [46]. We tailor on-demand well-selected arbitrary spatial polarization state distributions which represent spatially depolarized, i.e., unpolarized light.

We investigate classical polarization states of unpolarized light in the framework of Stokes parameters by exploiting a Cornu depolarizer for spatial depolarization combined with polarization state engineering with additional wave plates and polarization state characterization by spatially resolved Stokes parameter measurements. In order to generate spatially depolarized type II light, we exploit the spatial depolarization properties of an optically active Cornu depolarizer [47-49]. First, with only the Cornu depolarizer, we conclude from data analysis the generation of equator states of polarization, encompassing all linear polarization states on the Poincaré sphere's equator.

We then manipulate these equator states by applying various wave plate operations on these states. This is in the spirit of searching for quantum symmetries, such as $\mathrm{SU}(2)$ transformations, where here we now focus on the invariance properties of type II unpolarized light, providing a theoretical foundation using Stokes formalism [50] and experimental results for this particular type of light. Our exploitation of a Cornu depolarizer in combination with wave plates finally yields the realization of the full manifold of all generalized "great-circle states" on the Poincaré sphere, thus exhibiting full SU(2) transformation symmetry. This is proven via tomographic reconstruction of the great-circle states from spatially resolved polarization distributions that are obtained via Stokes parameter analysis. Furthermore, we reveal invariance properties of these great-circle states by systematically placing additional wave plates in the beam path with well-selected rotation angles. In the following, we describe the central concepts and our setup and develop a detailed theoretical model of these unpolarized states.

\section{TAILORING UNPOLARIZED LIGHT}

The key idea for manipulating unpolarized light states on the Poincaré sphere is in the spirit of polarization structuring or polarization engineering of light. The mathematical model for this is the Poincaré sphere, on which polarization states are usually visualized as single points on the sphere's surface for fully polarized light. Unpolarized light is depicted here in terms of areas, regions, distributions, or trajectories on the sphere. Here we investigate generalized great-circle states whose polarization distribution encompasses circles on the Poincaré sphere and whose spatially integrated total degree of polarization results in an overall value of zero, i.e., fully unpolarized light. Some examples of these generalized great-circle states are shown in Fig. 1 as solid circles. The generation, manipulation, and invariance properties of these polarization distributions will be in the focus of this paper. The experimental realization of these unpolarized light states will be discussed in the following section.

\section{EXPERIMENTAL SETUP AND METHODS}

\section{A. Setup}

The experimental setup is depicted in Fig. 2. Linearly $y$-polarized light from a helium-neon laser is emitted as a monochromatic, collimated, plane-wave beam, impinges on the Cornu depolarizer (CD) and fully illuminates its front facet. A half-wave plate (HWP) and two rotatable quarterwave plates $\left[\mathrm{QWP}_{1}(\alpha)\right.$ and $\mathrm{QWP}_{2}(\beta)$ ] placed behind the $\mathrm{CD}$ are then used to manipulate the polarization state of the output light. The transformed output light then enters a SchaeferCollett [51] or Berry-Gabrielse-Livingston [52] polarimeter, which consists of a rotatable quarter-wave plate $\left[\mathrm{QWP}_{\mathrm{pol}}(\theta)\right]$ and a fixed linear polarizer (LP). Finally, a spatially resolving CCD camera is used to investigate the light's polarization properties via spatially resolved Stokes parameter analysis. In previous work a similar setup was used, where the beam forming and sources of measurement uncertainties are discussed in detail [43]. Compared to this previous setup, notably, here the circular iris aperture in front of the $\mathrm{CD}$ is removed to have full spatial polarization dispersion. The core component of our setup is the $\mathrm{CD}$, which depolarizes the input light in the 


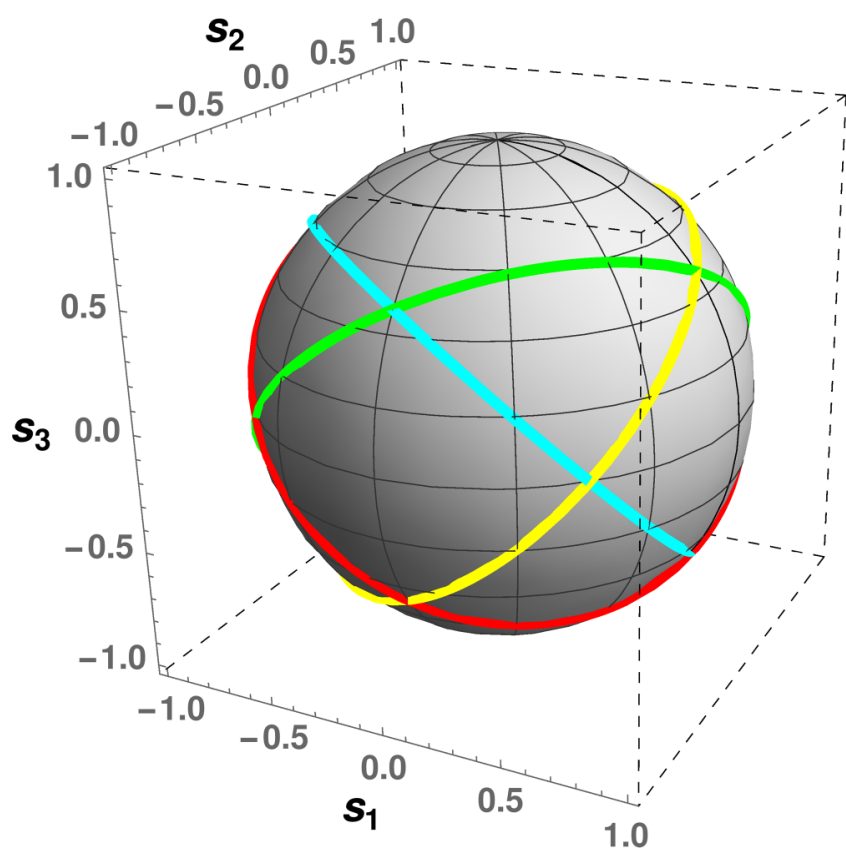

FIG. 1. Schematic depiction of exemplary generalized greatcircle states (shown as circles in green, yellow, red, and cyan) of unpolarized light on the Poincaré sphere, which is spanned by the three normalized Stokes vectors $s_{1}, s_{2}$, and $s_{3}$.

spatial domain and is illustrated in Fig. 2 on the left. The CD consists of two prism wedges consisting of uniaxial crystalline quartz joined at a slanted interface with a cut angle of $30^{\circ}$. One wedge consists of left-handed quartz and the other of right-handed quartz, with the fast axis of both wedges aligned in parallel to the direction of optical propagation. The circular birefringence causes an optical rotation of the polarization axis of linearly polarized input light, depending on a spatially dependent retardance $\Delta$.

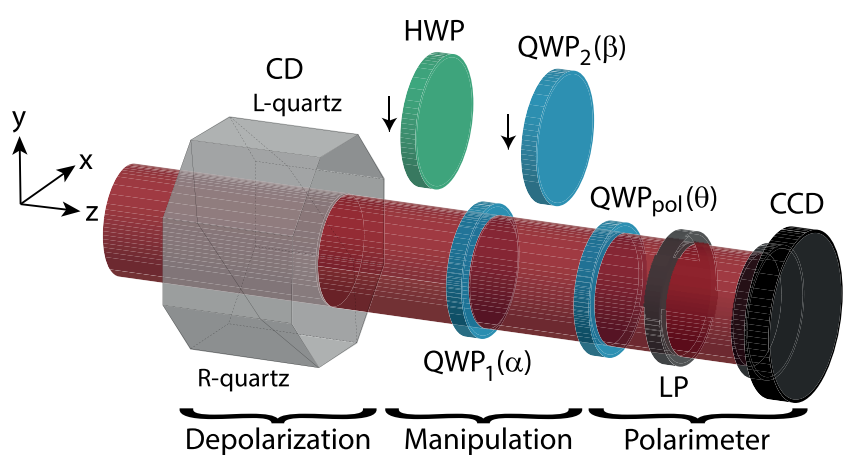

FIG. 2. Experimental setup: Cornu depolarizer (CD) followed by an optional half-wave plate (HWP), two rotatable quarter-wave plates $\left(\mathrm{QWP}_{1}\right.$ and $\mathrm{QWP}_{2}$, angles $\alpha$ and $\beta$ with respect to the $y$-axis), a polarimeter consisting of a rotatable quarter-wave plate $\left(\mathrm{QWP}_{\mathrm{pol}}\right)$, angle $\theta$ with respect to the LP), and linear polarizer (LP). A HWP and two subsequent rotatable QWPs are placed into the beam path depending on the desired output polarization state. A CCD camera acquires spatially resolved polarization properties.

\section{B. Methods}

In our experimental setup shown in Fig. 2, a CCD camera records spatially resolved intensity distributions for fixed angle settings of the imaging polarimeter. While the LP remains fixed, a $\mathrm{QWP}_{\mathrm{pol}}(\theta)$ is rotated in $10^{\circ}$ increments and for every angle position, a CCD image is taken. The data are then processed by performing Stokes parameter analysis using Fourier series [53] for every pixel of the CCD image and the normalized, spatially resolved Stokes parameters $s_{1}(x, y), s_{2}(x, y)$, and $s_{3}(x, y)$ are obtained. From these spatial normalized Stokes parameter distributions, we reconstruct tomographically the achieved unpolarized light states in the frame of the Poincaré sphere depiction (Figs. 5-9), the key result of the paper. For this purpose, we take a spatial coordinate pair (Pixel pair) $\left(x_{i}, y_{j}\right)$ on the CCD sensor and read out the corresponding values of $s_{1}, s_{2}$, and $s_{3}$, which are then plotted as a single point in $3 \mathrm{D}$ space spanned by $s_{1}, s_{2}$, and $s_{3}$. A small spatial binning procedure is performed to ensure a clear visual representation of the polarization distributions. The match (coincidence of the spatial coordinates) of the spatial orientation of $s_{1}, s_{2}$, and $s_{3}$ in space is extremely challenging, because a small offset between them creates the cloudy appearance visible in Figs. 5-9 being the major error source. This measuring procedure is repeated for various constellations of wave plates behind the $\mathrm{CD}$, which is accompanied by simulations of these polarization distributions, as will be discussed in the following.

\section{THEORETICAL FRAMEWORK}

In this section, we summarize our theoretical model for generating spatially unpolarized light states on the Poincaré sphere prior to the experimental investigations. The polarization properties of depolarized light are easily understood in the context of Stokes formalism, where the polarization state of light is quantified by a normalized four-valued vector $\vec{s}=\left(1, s_{1}, s_{2}, s_{3}\right)$ with its components representing linear and elliptical polarization parts [53]. Any modifications to this polarization state are then carried out by multiplying this vector with a series of Mueller matrices, which depend on the polarization optics placed in the beam path. Notably, the matrix of the $\mathrm{CD}$ is given as [54]

$$
M_{\mathrm{CD}}=\left(\begin{array}{cccc}
1 & 0 & 0 & 0 \\
0 & \cos (\Delta) & \sin (\Delta) & 0 \\
0 & -\sin (\Delta) & \cos (\Delta) & 0 \\
0 & 0 & 0 & 1
\end{array}\right)
$$

where $\Delta$ is a retardance that is induced by the birefringent wedges of the depolarizer that depends on material parameters and geometry of the CD [43].

For a linearly polarized input Stokes vector, the polarization axis is rotated about its propagation axis, depending on the retardance $\Delta$. Therefore, the CD redistributes both linear components $s_{1}$ and $s_{2}$, which translates into a polarization distribution that is known as a depolarized equator state, with Stokes vector components that are spread across the equator of the Poincaré sphere, thus spatially averaging to zero. 


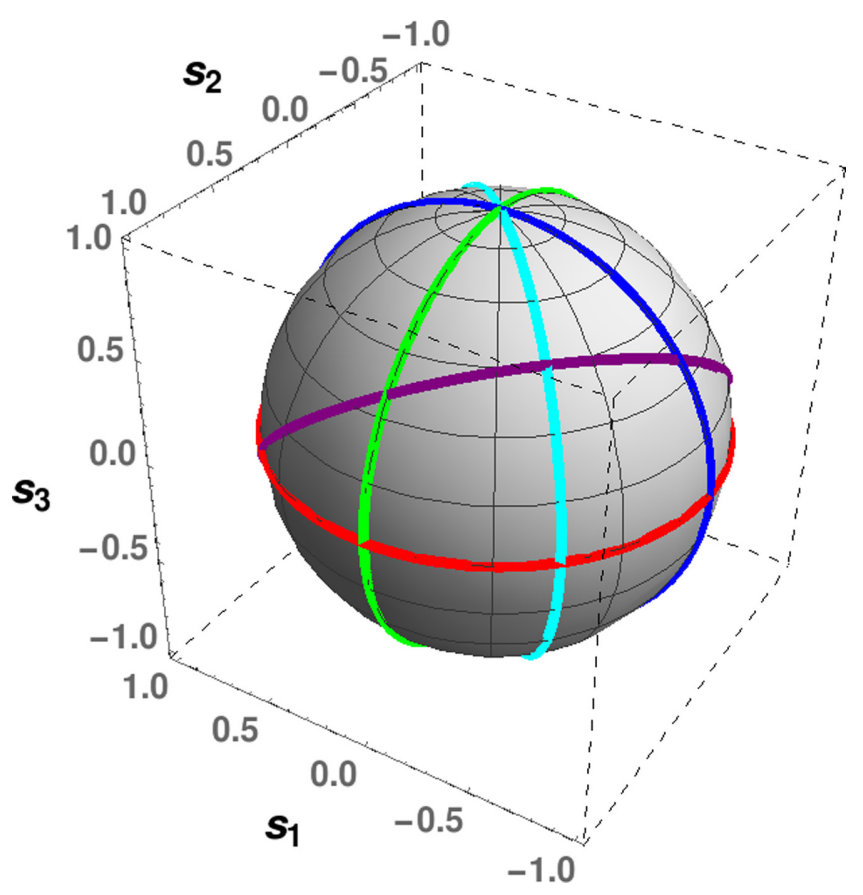

FIG. 3. Depiction of the Poincaré sphere, which is spanned by the three normalized Stokes vectors $s_{1}, s_{2}$, and $s_{3}$. Shown are an equator state (solid red circle), three meridian states for $\operatorname{QWP}_{1}(\alpha)$ rotation angles of $0^{\circ}$ (blue circle), $-22.5^{\circ}$ (cyan circle), and $-45^{\circ}$ (green circle) as well as a great-circle state $\left(\mathrm{QWP}_{1}\left(\alpha=0^{\circ}\right)\right.$ and $\mathrm{QWP}_{2}\left(\beta=67.5^{\circ}\right)$, magenta circle).

\section{A. Equator polarization states}

We begin by generating such an equator distribution of unpolarized light $P_{\text {eq }}$ from linearly $y$-polarized input light $P_{y}$, which is parallel to the $y$-axis shown in Fig. 2. An equator polarization distribution is produced by multiplying $P_{y}$ with the Mueller matrix of the CD:

$$
\begin{gathered}
P_{\mathrm{eq}}=M_{\mathrm{CD}} P_{y} \\
=\left(\begin{array}{cccc}
1 & 0 & 0 & 0 \\
0 & \cos (\Delta) & \sin (\Delta) & 0 \\
0 & -\sin (\Delta) & \cos (\Delta) & 0 \\
0 & 0 & 0 & 1
\end{array}\right)\left(\begin{array}{c}
1 \\
-1 \\
0 \\
0
\end{array}\right)=\left(\begin{array}{c}
1 \\
-\cos (\Delta) \\
\sin (\Delta) \\
0
\end{array}\right) .
\end{gathered}
$$

This equator state is shown in Fig. 3 as a solid red circle encompassing the entire equator. For the investigation of the invariances of this polarization state, we then simulate the insertion of a HWP placed into the beam behind the CD with a suitable Mueller matrix. Setting the HWP to an arbitrary rotation angle leaves the distribution's position unchanged and results in another equator distribution $P_{\text {eq }}^{\prime}$, which indicates that the equator distribution's position is invariant with respect to an HWP. Here the HWP's influence on the polarization distribution is equivalent to a rotation about the $s_{3}$-axis.

\section{B. Meridian polarization states}

We now proceed with tailoring or manipulating these equator states into meridian polarization states, by removing the HWP and inserting a $\mathrm{QWP}_{1}(\alpha)$ into the beam behind the
$\mathrm{CD}$ and observe the change of the polarization state as the angle $\alpha$ is varied. The generation of these meridian states on the Poincare sphere is now modeled by multiplying the appropriate Mueller matrix of a rotated $\mathrm{QWP}_{1}$ (rotation angle $\alpha)$ with $P_{\text {eq }}$ :

$$
P_{m}=M_{\mathrm{QWP}_{1}}(\alpha) P_{\mathrm{eq}} .
$$

These polarization distributions are distinguished by the fact that their position is perpendicular to the equator plane. For illustrative purposes, three $\mathrm{QWP}_{1}$ rotation angles of $0^{\circ},-22.5^{\circ}$, and $-45^{\circ}$ are chosen in the following to generate distinct meridian states. We start by calculating a "prime meridian" [55] distribution with a $\mathrm{QWP}_{1}$ rotation angle of $0^{\circ}$ :

$$
P_{m, 0^{\circ}}=M_{\mathrm{QWP}_{1}}\left(0^{\circ}\right) P_{\mathrm{eq}}=\left(\begin{array}{c}
1 \\
-\cos (\Delta) \\
0 \\
\sin (\Delta)
\end{array}\right) .
$$

Comparing Eq. (5) with Eq. (3), the intensity of $s_{2}$ is reduced to zero, while the meridian state consists of $s_{1}$ and $s_{3}$ components with a sinusoidal distribution with respect to the retardance $\Delta$. This prime meridian distribution (equivalent to the "Greenwich" meridian in geodetic terms) [55] is indicated by a blue circle in Fig. 3 which encompasses the sphere.

Decreasing the $\mathrm{QWP}_{1}$ angle $\alpha$ further to $-22.5^{\circ}$, the following polarization distribution is obtained:

$$
\begin{aligned}
& P_{m,-22.5^{\circ}}=M_{\mathrm{QWP}_{1}}\left(-22.5^{\circ}\right) P_{\mathrm{eq}} \\
& =\left(\begin{array}{c}
1 \\
-\frac{1}{2} \cos (\Delta)-\frac{1}{2} \sin (\Delta) \\
\frac{1}{2} \cos (\Delta)+\frac{1}{2} \sin (\Delta) \\
-\frac{1}{\sqrt{2}} \cos (\Delta)+\frac{1}{\sqrt{2}} \sin (\Delta)
\end{array}\right) .
\end{aligned}
$$

In Fig. 3 this polarization distribution ("São Paulo") [55] is shown as a cyan circle. Here the components of the spatial Stokes parameters are redistributed such that $s_{1}(x, y)$ and $s_{2}(x, y)$ are equal with opposite sign, with their amplitude reduced by $1 / 2$ in comparison with the $s_{1}$ component of the $P_{m, 0^{\circ}}$ distribution. Compared to the $s_{3}(x, y$,$) component of the$ previous distribution $P_{m, 0^{\circ}}$ in Eq. (5), the distribution $s_{3}(x, y$, is shifted by $\pi / 4$.

Decreasing the $\mathrm{QWP}_{1}$ angle even further to $-45^{\circ}$, the polarization distribution of the third meridian state ("New Orleans") [55] is obtained:

$$
P_{m,-45^{\circ}}=M_{\mathrm{QWP}_{1}}\left(-45^{\circ}\right) P_{\mathrm{eq}}=\left(\begin{array}{c}
1 \\
0 \\
\sin (\Delta) \\
-\cos (\Delta)
\end{array}\right) \text {. }
$$

In this polarization distribution, the $s_{1}(x, y)$ component vanishes and the Stokes vector $\vec{s}$ now consists of sinusoidal $s_{2}(x, y)$ and cosinusoidal $s_{3}(x, y)$ components and its representation is visualized by a green circle in Fig. 3 . These selected polarization distributions of the meridian states are illustrated in Fig. 3 as continuous circles extending across the Poincare sphere's surface. The three particular $\mathrm{QWP}_{1}(\alpha)$ rotation angles of $0^{\circ},-22.5^{\circ}$, and $-45^{\circ}$ were chosen to illustrate the positioning of the meridian states depending on $\alpha$. The distribution $P_{90^{\circ}}$ is equivalent to $P_{0^{\circ}}$ due to rotational 
symmetry. This behavior can be understood in analogy to how a QWP acts upon linearly polarized light. Now the invariance of the meridian states with respect to phase changes is checked by inserting additional wave plates behind the first $\mathrm{QWP}_{1}$. Inserting a HWP behind the $\mathrm{QWP}_{1}(\alpha)$ and calculating the resulting distributions for various HWP angles indicates that the meridian states are rotated about the $s_{3}$-axis yet again, with a HWP angle of $-90^{\circ}$ transforming the prime meridian onto itself:

$$
M_{\mathrm{HWP}}\left(-90^{\circ}\right) P_{m, 0^{\circ}}=P_{m, 0^{\circ}} .
$$

After these successful modeling predictions of the meridian states we now proceed to the generalized great-circle states.

\section{Generalized great-circle polarization states}

Finally, inserting an additional $\mathrm{QWP}_{2}$ (rotation angle $\beta$ ) after the first $\mathrm{QWP}_{1}$, enables the generation of "tilted" meridian states, i.e., meridian polarization distributions on the Poincare sphere that are rotated about an axis that lies in the plane spanned by $s_{1}$ and $s_{2}$. Some of these exemplary generalized great-circle states are shown schematically in Fig. 1. Borrowing from nomenclature in geodesy, we refer to these as distributions as generalized "great-circle states." The generation of these generalized great-circle states $P_{g c}$ is accomplished similarly using appropriate Mueller matrices.

We present a prominent example of a generalized greatcircle state by choosing a rotation angle of $\beta=67.5^{\circ}$ to generate it from a prime meridian state [Eq. (5)]:

$$
\begin{aligned}
& P_{g c, 67.5^{\circ}}=M_{\mathrm{QWP}_{2}}\left(67.5^{\circ}\right) P_{m, 0^{\circ}} \\
& =\left(\begin{array}{c}
1 \\
-\frac{1}{2} \cos (\Delta)+\frac{1}{\sqrt{2}} \sin (\Delta) \\
\frac{1}{2} \cos (\Delta)+\frac{1}{\sqrt{2}} \sin (\Delta) \\
\frac{1}{\sqrt{2}} \cos (\Delta)
\end{array}\right) .
\end{aligned}
$$

This distribution contains contributions in all three Stokes parameters and is displayed in Fig. 3 as magenta circle on the Poincaré sphere.

Since these generalized great-circle states are mathematically derived from the meridian states, we calculate two extreme cases where the generalized circle states coincide with previously shown polarization distributions.

In the first example, we start with a prime meridian state and set a second $\mathrm{QWP}_{2}$ to $\beta=0^{\circ}$, which produces an equator state [cf. Eq. (3)]:

$$
P_{g c, 0^{\circ}}=M_{\mathrm{QWP}_{2}}\left(0^{\circ}\right) P_{m, 0^{\circ}}=P_{\mathrm{eq}} .
$$

In the second example, setting the $\mathrm{QWP}_{2}(\beta)$ rotation angle to $-45^{\circ}$ transforms the prime meridian state back into another prime meridian state:

$$
P_{g c,-45^{\circ}}=M_{\mathrm{QWP}_{2}}\left(-45^{\circ}\right) P_{m, 0^{\circ}}=P_{m, 0^{\circ}} .
$$

Inspecting the great-circle states distribution's position and tilt angle with respect to the Poincaré sphere's equatorial plane as $\beta$ is varied reveals two aspects of the great-circle states: first, the two points, where the great-circle states distributions intersect with the equator, are rotated about the $s_{3}$-axis as $\beta$ is varied. Second, the tilt angle of the distribution also depends on the angle $\beta$.
Finally, we probe the invariance properties of the greatcircle states by placing a single additional HWP behind both QWPs $\left(\mathrm{QWP}_{1}\right.$ and $\left.\mathrm{QWP}_{2}\right)$ and varying the HWP's rotation angle for fixed rotation angles of the QWPs. This produces a similar result as the previous wave-plate constellation consisting of $\mathrm{QWP}_{1}(\alpha)$ and a HWP (cf. Sec. IV B): as the HWP angle is increased, the generalized great-circle states are rotated about the $s_{3}$-axis. This shows that the generalized great-circle states are not invariant with respect to the phase change of a HWP.

In the following section, we now present experimental results, which confirm the generation of the previously presented simulations of the polarization distributions and their invariance properties.

\section{EXPERIMENTAL RESULTS}

\section{A. Equator polarization states}

First, we verified the imaging quality of our setup without the $C D$ to ensure that a Gaussian intensity distribution is maintained throughout. Next, we investigated the polarization-dispersed output light behind the $\mathrm{CD}$ as shown in the experimental setup in Fig. 2, i.e., without any wave plates between depolarizer and polarimeter. Using the polarimeter, the spatial polarization distributions of the Stokes parameters $s_{1}(x, y), s_{2}(x, y)$, and $s_{3}(x, y)$ were obtained from images recorded by the CCD camera. We measured characteristic polarization fringe patterns schematically represented by the spatial Stokes parameter distributions shown in Fig. 4, which could be explained conclusively within the Mueller matrix formalism [43]. The granular structure shown in Fig. 4 is partly due to the error bars introduced by imperfect wave plates (off-axis spatial inhomogeneuity), the beam quality, and the CCD's noise floor.

The position of the polarization fringe pattern with respect to the $y$-axis as well as the contrast of the $s_{i}(x, y)$ values in these spatial Stokes parameter distributions is manipulated, depending on the constellation of wave plates between the CD and the polarimeter, which we call polarization engineering. From the polarization fringe patterns, their change in position and the contrast, we are able to tomographically reconstruct the spatial polarization distributions for every constellation.

Here we now present from these spatial Stokes parameter distributions, a tomographic reconstruction of an equator state and obtain a red annular ringlike polarization distribution in Fig. 5 that is slightly skewed due to measurement errors, but still closely resembles the theoretically calculated and expected equator state calculated in Eq. (3). Finally, placing a HWP between the CD and the polarimeter revealed an invariance of these polarization distributions for arbitrary rotation angles of the HWP, which implies that in fact we produced type II unpolarized light [42].

\section{B. Meridian polarization states}

We then proceed by replacing the HWP with a rotatable $\mathrm{QWP}_{1}(\alpha)$ between the $\mathrm{CD}$ and polarimeter to produce the meridian states. The spatial distributions of the Stokes parameters shown in Fig. 4, which we obtained from images of the CCD camera placed behind the polarimeter in Fig. 2, display 


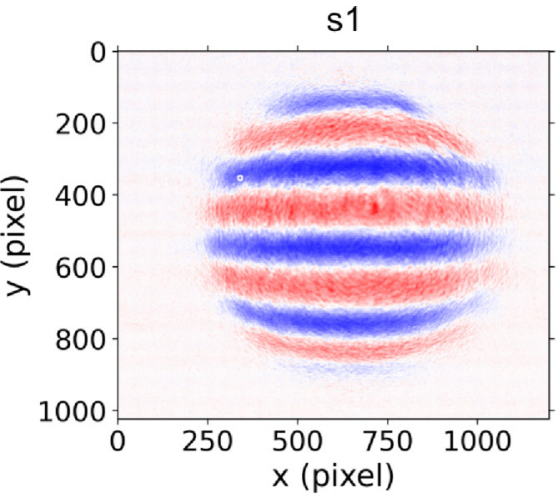

s2

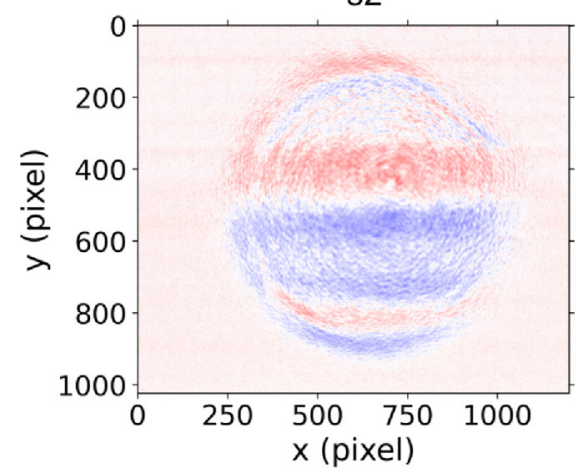

s3

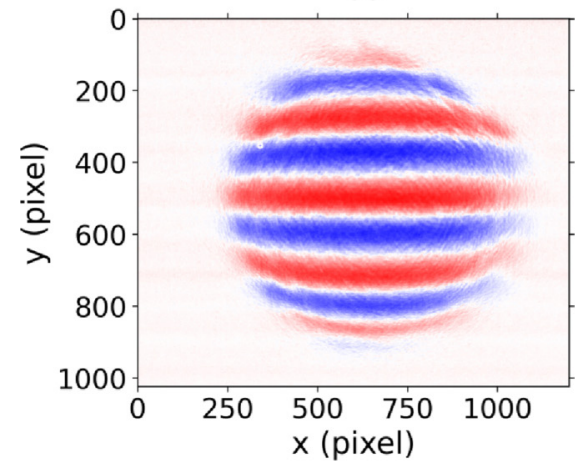

s1

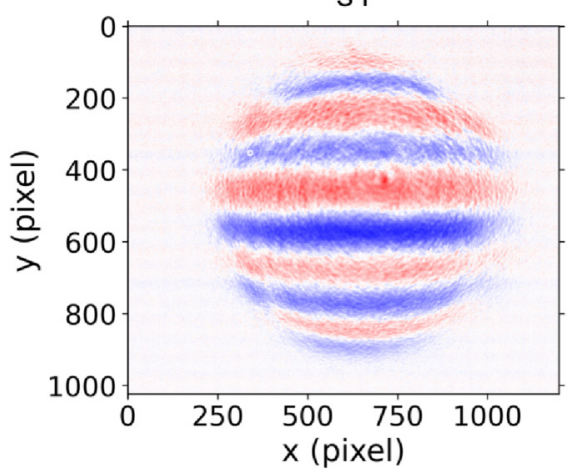

s2

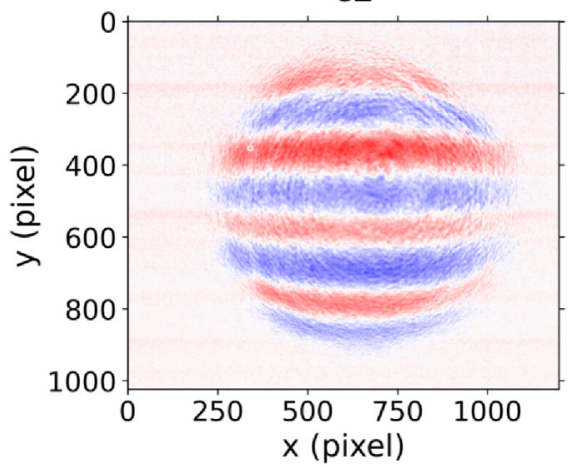

s3

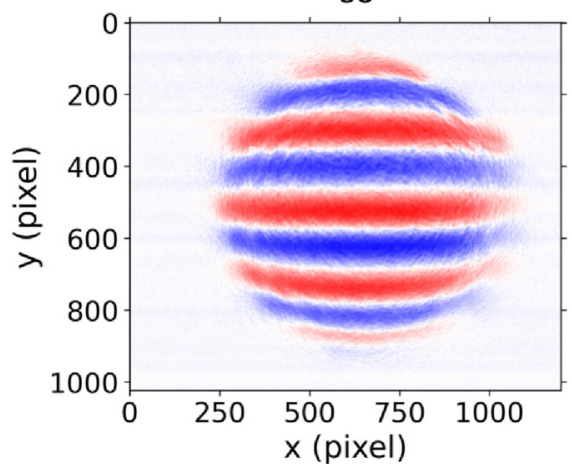

s1

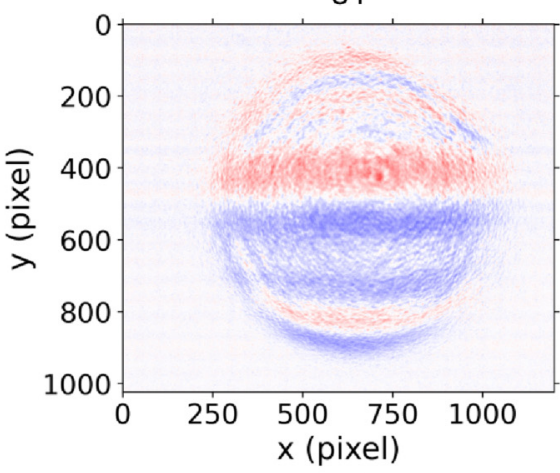

s2

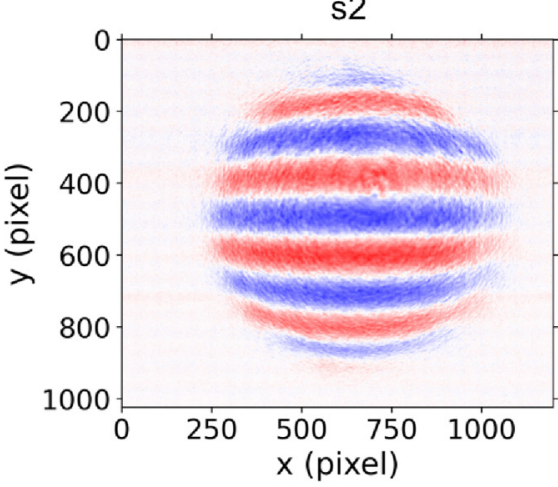

s3

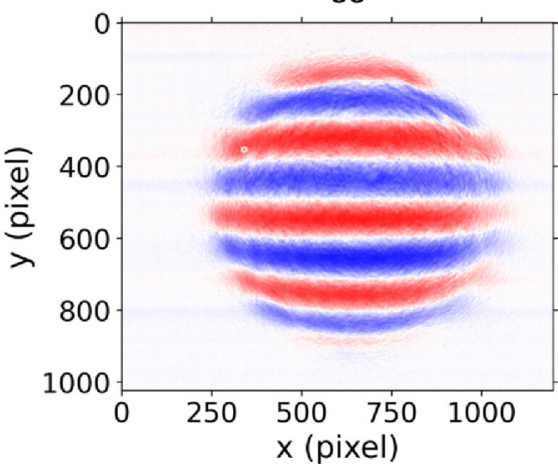

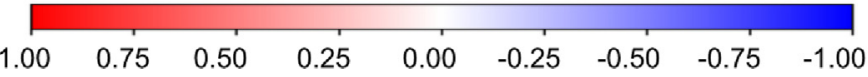

FIG. 4. Spatial distribution of the normalized Stokes parameters $s_{1}(x, y)$ (first row), $s_{2}(x, y)$ (second row), and $s_{3}(x, y)$ (third row) obtained

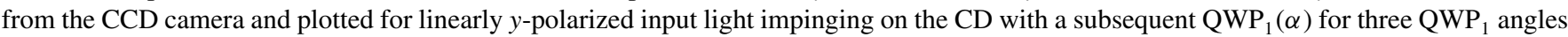
of $0^{\circ}$ (first column), $-22.5^{\circ}$ (second column), and $-45^{\circ}$ (third column), representing three prominent meridian states. The $x$ - and $y$-axes are scaled in terms of the pixel number of the CCD. Red indicates positive values, blue indicates negative values. A small degree of barrel distortion is visible around the profile's edges.

evenly spaced characteristic polarization fringes where the Stokes parameters range between values of -1 and 1 . We demonstrate the generation of the three previously calculated meridian states for three distinct $\mathrm{QWP}_{1}(\alpha)$ rotation angles of $0^{\circ},-22.5^{\circ}$, and $-45^{\circ}$.

For a $\mathrm{QWP}_{1}$ rotation angle of $0^{\circ}$ (left column in Fig. 4), the spatial distributions of $s_{1}(x, y)$ and $s_{3}(x, y)$ are dominated by broad polarization fringes, while they are almost absent in the distribution of $s_{2}(x, y)$. In the Poincaré sphere model, this set of $s_{i}(x, y)$ distributions corresponds to the prime meridian state. This correspondence becomes clearly apparent in the subsequent tomographic reconstructions of these polarization states.
When comparing the distributions of $s_{1}(x, y)$ of all three meridian states (top row in Fig. 4), a high contrast of 2 for its values is observed for the $s_{1}(x, y)$-distribution of the prime meridian in the left column. This contrast decreases as the $\mathrm{QWP}_{1}$ angle is set to $0^{\circ},-22.5^{\circ}$, and finally $-45^{\circ}$, where the fringe contrast reaches a minimum value of roughly 0.5 . For the $s_{2}(x, y)$ distributions in the second row, an opposite trend emerges: the contrast is lowest for a $\mathrm{QWP}_{1}$ angle of $0^{\circ}$, increases with the decreasing QWP angle and reaches its maximum value of 2 for a $\mathrm{QWP}_{1}$ angle of $-45^{\circ}$. This redistribution of the $s_{1}(x, y)$ and $s_{2}(x, y)$ values is proof of the meridian state being rotated about the $s_{3}$-axis. For all three $\mathrm{QWP}_{1}$ rotation angles, the $s_{3}(x, y)$-distributions in the third 


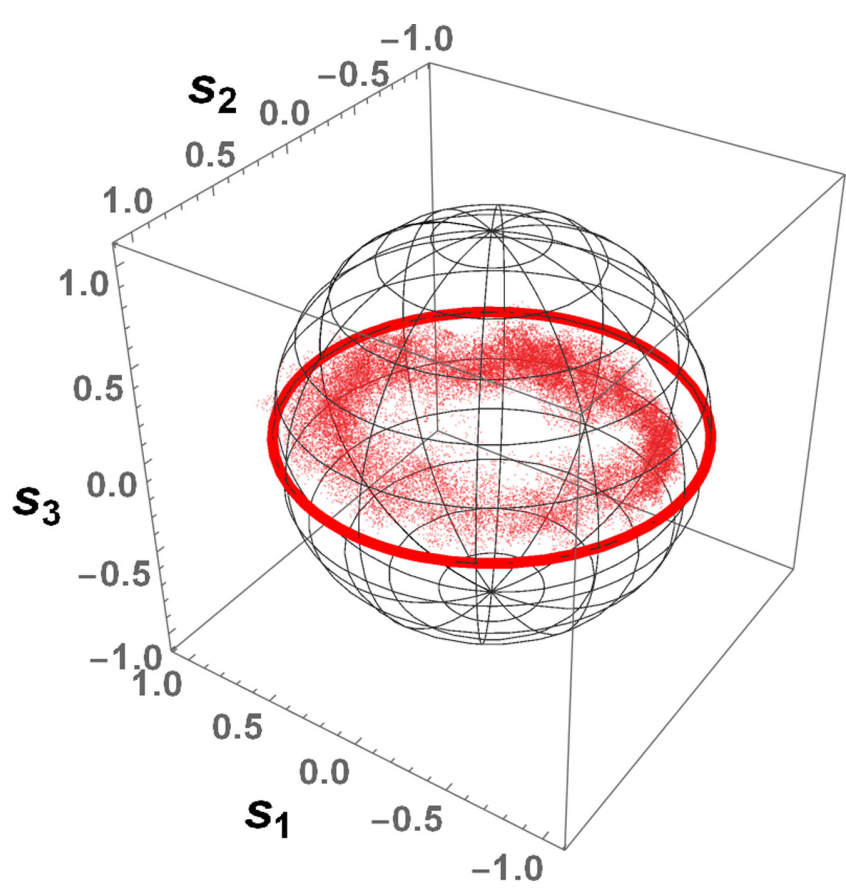

FIG. 5. Equator distribution generated by only the CD: the spatial Stokes parameter distribution of the corresponding CCD polarization distributions was mapped to the Poincaré sphere and is indicated by a red annular ring. The slight tilt is a due to measurement errors. A corresponding simulation of the equator state is shown as a solid red circle.

row exhibit a polarization fringe pattern, which is spatially shifted along the $y$-axis by $\pi / 4$ when setting the $\mathrm{QWP}_{1}$ angle from $0^{\circ}$ to $-22.5^{\circ}$ and shifted once again by $\pi / 4$, when setting it to $-45^{\circ}$. This spatial shift is also verified by our previous simulations of all three polarization distributions.

To better visualize the computed Stokes polarization vectors for each pixel of the recorded CCD images, we show them on the Poincare sphere to see the distribution of the sets of the Stokes vectors. Therefore, we take a rectangular shaped area around a defined center of the beam in the CCD images. The rectangle has a width of 256 pixels wide and length of 128 pixels, resulting in 32768 dedicated Stokes vectors, which are then plotted on the Poincaré sphere.

As a result, the annular rings are formed by mapping the $s_{i}(x, y)$ distributions corresponding Stokes vectors on the Poincaré sphere, thus representing a tomographic reconstruction of a meridian state. Utilizing this method, the spatial polarization distributions of the Stokes parameters shown in Fig. 4 are also visualized as red annular rings in Fig. 6, Fig. 7, and Fig. 8 for $\mathrm{QWP}_{1}$ rotation angles of $0^{\circ},-22.5^{\circ}$, and $-45^{\circ}$, together with simulated distributions indicated in colors (blue, cyan, and green), respectively. It is noteworthy that due to some remaining residual polarization of the input beam, the Stokes vectors always have a value smaller than one. This residual polarization is transferred through the entire beam propagation path and causes error propagation, which in return results in the cloudlike appearance of the polarization distributions.

In Fig. 6 both the annular ringlike distribution of the Stokes vectors encompassing the Poincaré sphere and its orientation

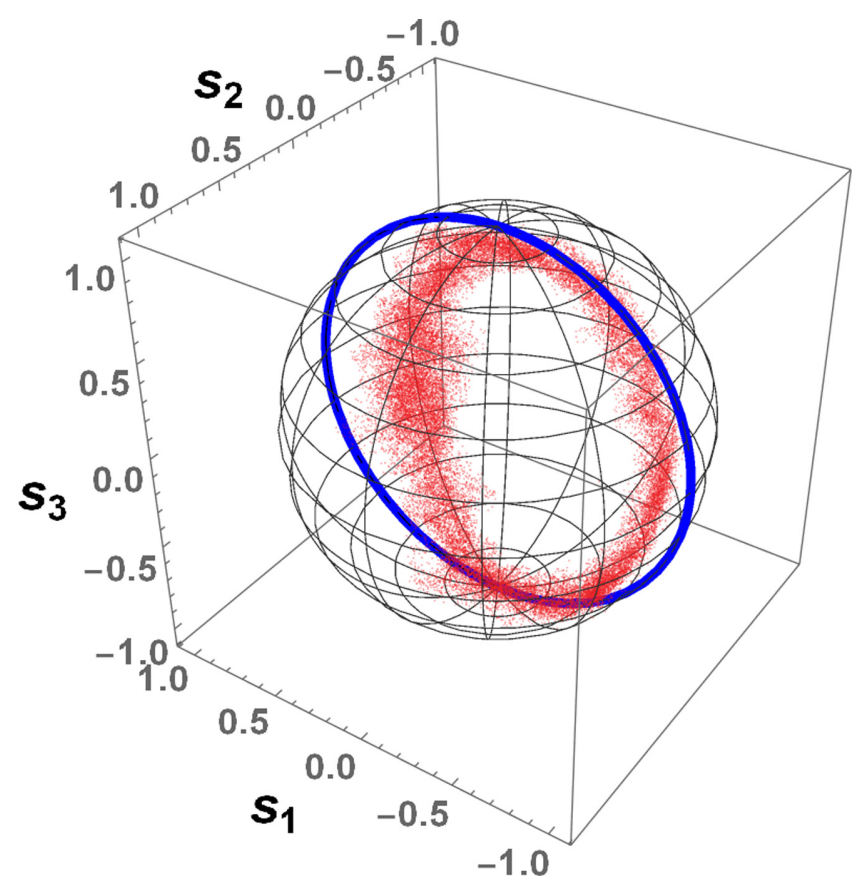

FIG. 6. Prime meridian state distribution generated by $\mathrm{QWP}_{1}\left(\alpha=0^{\circ}\right)$ : the Stokes parameter distributions of the first column of Fig. 4 are mapped to the Poincaré sphere and indicated by a red annular ring. The corresponding simulation is shown as a solid blue circle.

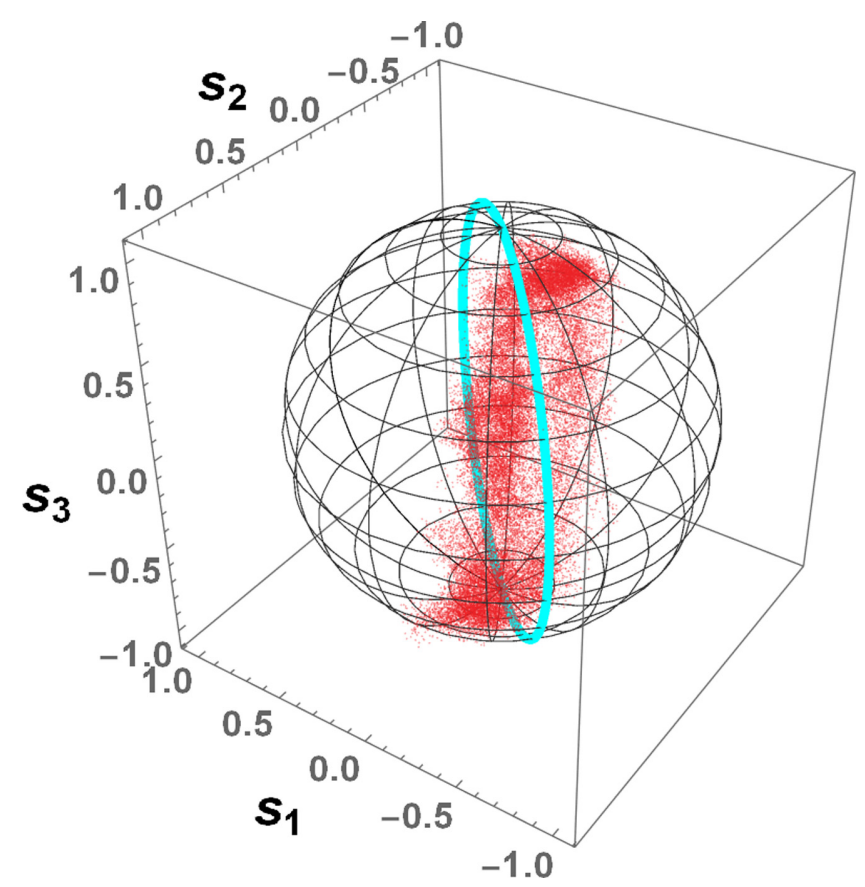

FIG. 7. Meridian state distribution generated by $\operatorname{QWP}_{1}(\alpha=$ $\left.-22.5^{\circ}\right)$ : the Stokes parameter distributions of the second column of Fig. 4 are mapped to the Poincaré sphere and indicated by a red annular ring. The corresponding simulation is shown as a solid cyan circle. 


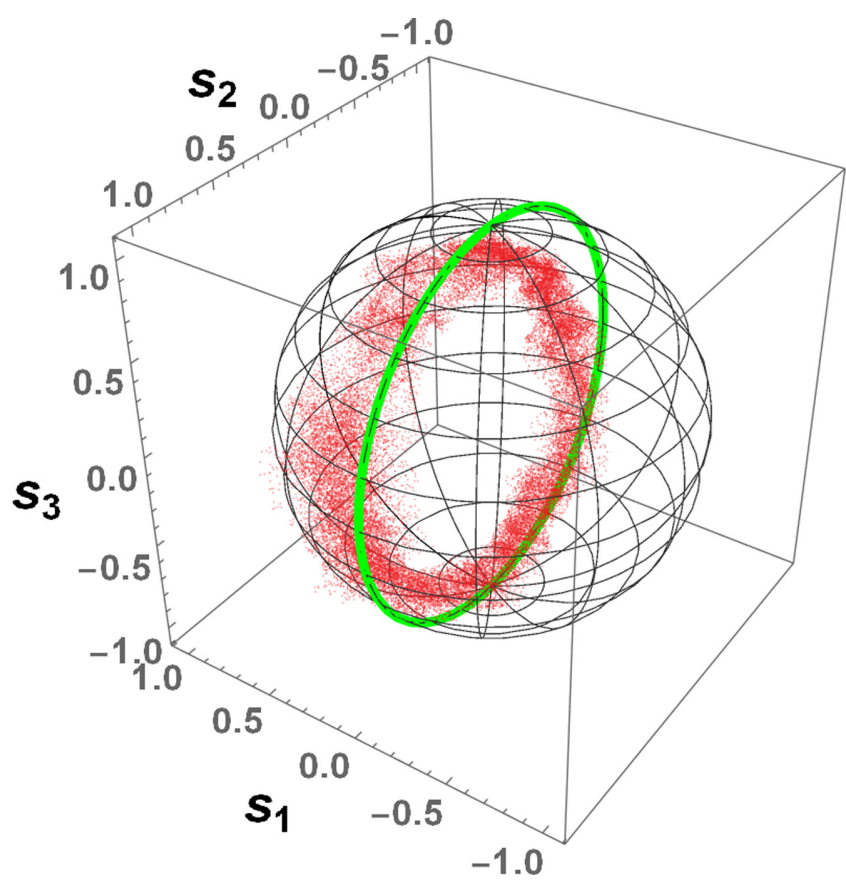

FIG. 8. Meridian state distribution generated by $\mathrm{QWP}_{1}(\alpha=$ $\left.-45^{\circ}\right)$ : the Stokes parameter distributions of the third column of Fig. 4 are mapped to the Poincaré sphere and indicated by a red annular ring. The corresponding simulation is shown as a solid green circle.

agree with the previous ring-shaped simulation of the prime meridian in Eq. (5). In Fig. 7 the distribution for a $\mathrm{QWP}_{1}$ rotation angle of $-22.5^{\circ}$ is slightly skewed due to small misalignment errors of the wave plates, but the rotation with respect to the $s_{3}$-axis is clearly evident.

Finally, we then investigated the invariance properties of the meridian states, by starting with a prime meridian $\left[\mathrm{QWP}_{1}\left(\alpha=0^{\circ}\right)\right]$ and subsequently introduced a HWP behind this QWP. We observed the polarization distributions for various HWP rotation angles in the same manner as before. Under the HWP's influence, the $s_{1}(x, y)$ and $s_{2}(x, y)$ components of the polarization distributions change in accordance with the simulated distributions, indicating a rotation of the polarization distribution about the $s_{3}$-axis. This proves that the meridian states are not invariant with respect to a phase change by a HWP (as opposed to the equator states). This invariance has to be explained by a novel state of unpolarized light, namely, type III unpolarized light.

\section{Generalized great-circle polarization states}

After having achieved equator and meridian distributions, we introduce the generalized great-circle states. For the generation of these, we placed a second $\mathrm{QWP}_{2}(\beta)$ behind the first $\mathrm{QWP}_{1}(\alpha)$. Setting the first $\mathrm{QWP}_{1}$ to a fixed angle of $\alpha=0^{\circ}$ and rotating the second $\mathrm{QWP}_{2}(\beta)$ to angles of $\beta=0^{\circ}$, $\beta=-22.5^{\circ}$, and $\beta=-45^{\circ}$, respectively, we observe polarization distributions that are still essentially uniform rings encompassing the sphere. For $\beta=-22.5^{\circ}$, the polarization distribution is tilted about an axis which is located in the equatorial plane spanned by $s_{1}$ and $s_{2}$. Similarly, spatial po-

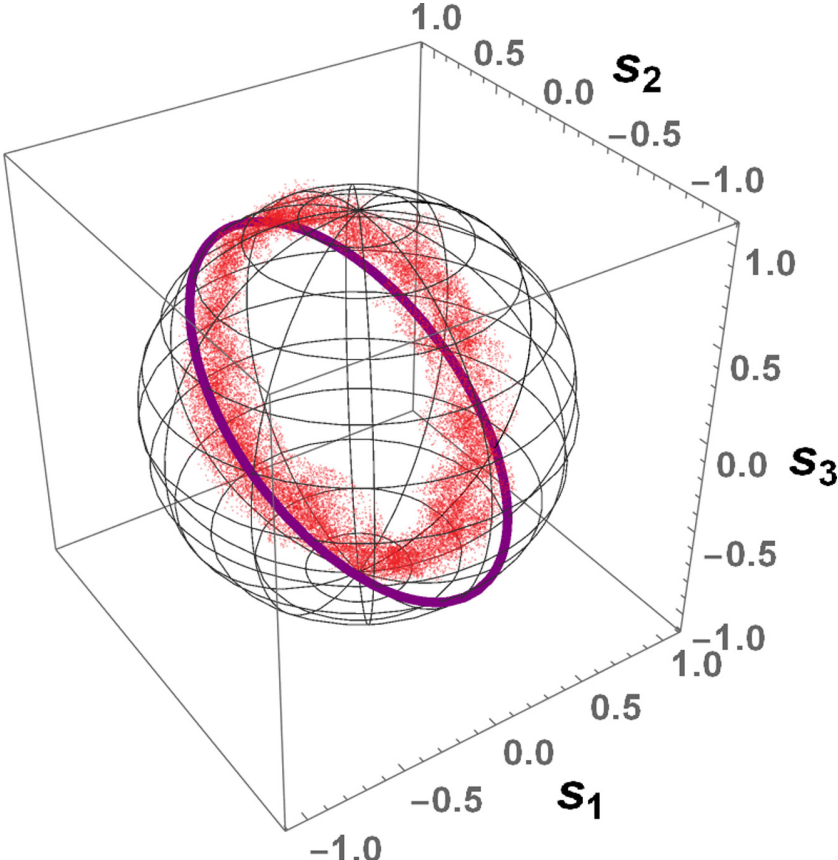

FIG. 9. Great-circle distribution generated by $\mathrm{QWP}_{1}\left(\alpha=0^{\circ}\right)$ followed by $\mathrm{QWP}_{2}\left(\beta=67.5^{\circ}\right)$ : the Stokes parameter distributions of the corresponding CCD polarization distributions were mapped to the Poincaré sphere and are indicated by a red annular ring. The corresponding simulation is shown as a solid magenta circle.

larization distributions obtained from the spatially resolved CCD images were mapped to the Poincaré sphere and a distinguished great-circle distribution is shown in Fig. 9 for a $\mathrm{QWP}_{2}$ rotation angle of $\beta=67.5^{\circ}$. We chose this specific angle to illustrate the visible tilt of the polarization distribution, since for angles of $\beta=0^{\circ}$ and $\beta=-45^{\circ}$, an equator distribution and a prime meridian distribution are obtained, respectively. These two polarization distributions are also shown in Fig. 3 as red circle (equator) and blue circle (meridian).

In Fig. 9 the distribution is tilted about roughly $45^{\circ}$ with respect to the $\left(s_{1}, s_{2}\right)$-plane, which is in agreement with the distribution given in Eq. (11).

In conclusion, this proves that we have not only generated type II unpolarized light, but were also able to control the resulting polarization distribution's position on the Poincaré sphere by manipulating rotation angles of two quarter-wave plates placed behind the $\mathrm{CD}$.

\section{DISCUSSION}

The simulations of the polarization distributions of unpolarized light are in excellent agreement with the experimental spatially resolved polarization distributions obtained from CCD images, which clearly demonstrate a systematic and controlled generation of these distributions of type II and type III unpolarized light.

In the first case, this is confirmed by the invariance of the position of the equator states when a HWP is placed behind the $\mathrm{CD}$. In the second case, the meridian states generated by a $\operatorname{QWP}_{1}(\alpha)$ are similar to uniform-banded distributions of type III unpolarized light, which was first proposed by 
Ellis et al. [39] in theory as a subclass of type II unpolarized light. Introducing a subsequent HWP rotates the position of the meridian states about the $s_{3}$-axis, which means that this type of light is not invariant with respect to phase changes of a HWP, which confirms that we have generated type III unpolarized light. Finally, the $\mathrm{CD}$ in conjunction with two subsequently following QWPs enables the full range of possible rotation transformations of the great circles of unpolarized light on the Poincaré sphere. This shows that discernible internal polarization states of unpolarized light are accessible by simply placing wave plates behind a CD. By doing this, we are able to elegantly generate on-demand unpolarized states by rotating phase plates to specific angles and are able to inspect their invariance properties. This Poincaré sphere polarization engineering allows realizing on-demand depolarized and delocalized polarization states distributed over the Poincaré sphere in well-selected areas or regions and complement the classical and quantum understanding of polarization with interesting application aspects.

In combination with a variable iris aperture placed behind the $\mathrm{CD}$, a broad tuning region of partially polarized and fully unpolarized type II light is accessible [43], depending on the aperture diameter and the rotation angles of the wave plates behind the depolarizer. This tuning range for type II unpolarized light is of particular significance in the context of Floquet engineering [23,24].

\section{CONCLUSION}

We have demonstrated the experimental generation of "equator states," "meridian states," and arbitrarily positioned "great-circle states" of unpolarized light by exploiting the spatial depolarization properties of a Cornu depolarizer in combination with two subsequent quarter-wave plates. We have presented a robust method of tailoring type II unpolarized light on the Poincaré sphere, together with a high level of control over these polarization distributions by varying the rotation angles of both quarter-wave plates.

The experimental results are in excellent agreement with a theoretical description of these polarization distributions within the Stokes parameter formalism in combination with Mueller matrix calculus. Furthermore, the polarization distributions displayed certain invariance properties with respect to the phase plates, which were explained in theory and are supported by experimental data. These findings serve as a vantage point for future research in the field of polarization correlations of unpolarized light and metrology of hidden polarization states. Notably, the polarization correlations of type I temporally unpolarized light and type II spatially unpolarized light and cross-correlations between various types of unpolarized light will reveal further characteristics of unpolarized light and possibly pave the way for precisely tailored depolarized states of light with a subsequent exploitation in metrology and imaging applications.

The data that support the findings of this study are available from the corresponding author upon reasonable request.

\section{ACKNOWLEDGMENTS}

The authors thank Andreas Herdt for fruitful and stimulating discussions and experimental support and Th. Führer for the loan of a CCD camera. We gratefully acknowledge discussions on the CD with Dr. Götz Zinner from B. Halle Nachfl. GmbH (Berlin) and the competent and innovative craftsmanship of this extraordinary optical manufacture. We thank Prof. A. T. Friberg for stimulating discussions about polarization and for two wonderful conferences on "Coherence and Polarization." The authors acknowledge funding from the Deutscher Akademischer Austauschdienst ("Breakthroughs in Ghost Imaging") and Deutsche Forschungsgemeinschaft (EL 105-21).
[1] A. M. Beckley, T. G. Brown, and M. A. Alonso, Full Poincaré beams, Opt. Express 18, 10777 (2010).

[2] L. Du, Z. Man, Y. Zhang, C. Min, S. Zhu, and X. Yuan, Manipulating orbital angular momentum of light with tailored in-plane polarization states, Sci. Rep. 7, 41001 (2017).

[3] C. Samlan and N. K. Viswanathan, Generation of vector beams using a double-wedge depolarizer: Non-quantum entanglement, Optics Lasers Eng. 82, 135 (2016).

[4] Y. Shen, Z. Wang, X. Fu, D. Naidoo, and A. Forbes, SU(2) Poincaré sphere: A generalized representation for multidimensional structured light, Phys. Rev. A 102, 031501(R) (2020).

[5] A. Forbes, M. de Oliveira, and M. R. Dennis, Structured light, Nat. Photonics 15, 253 (2021).

[6] A. Forbes, Structured light from lasers, Laser Photonics Rev. 13, 1900140 (2019).

[7] A. Z. Goldberg, P. de la Hoz, G. Björk, A. B. Klimov, M. Grassl, G. Leuchs, and L. L. Sánchez-Soto, Quantum concepts in optical polarization, Adv. Opt. Photonics 13, 1 (2021).

[8] S. Said and N. L. Bihan, Higher-order statistics of Stokes parameters in a random birefringent medium, Waves Random Complex Media 18, 275 (2008).
[9] U. Schilling, J. von Zanthier, and G. S. Agarwal, Measuring arbitrary-order coherences: Tomography of single-mode multiphoton polarization-entangled states, Phys. Rev. A 81, 013826 (2010).

[10] R. Simon and N. Mukunda, Minimal three-component SU(2) gadget for polarization optics, Phys. Lett. A 143, 165 (1990).

[11] V. Bagini, R. Borghi, F. Gori, M. Santarsiero, F. Frezza, G. Schettini, and G. S. Spagnolo, The Simon-Mukunda polarization gadget, Eur. J. Phys. 17, 279 (1996).

[12] Á. Rivas and A. Luis, SU(2)-invariant depolarization of quantum states of light, Phys. Rev. A 88, 052120 (2013).

[13] S. G. Reddy, S. Prabhakar, A. Aadhi, A. Kumar, M. Shah, R. P. Singh, and R. Simon, Measuring the Mueller matrix of an arbitrary optical element with a universal $\mathrm{SU}(2)$ polarization gadget, J. Opt. Soc. Am. A 31, 610 (2014).

[14] S. G. Reddy, S. Prabhakar, P. Chithrabhanu, R. P. Singh, and R. Simon, Polarization state transformation using two quarter wave plates: Application to Mueller polarimetry, Appl. Opt. 55, B14 (2016).

[15] M. Bass, C. DeCusatis, J. Enoch, V. Lakshminarayanan, G. Li, C. Macdonald, V. Mahajan, and E. Van Stryland, Handbook of 
Optics, Volume II: Design, Fabrication and Testing, Sources and Detectors, Radiometry and Photometry, 3rd ed. (McGraw-Hill, New York, 2009).

[16] H. Hurwitz, The statistical properties of unpolarized light, J. Opt. Soc. Am. 35, 525 (1945).

[17] A. Langsdorf and L. A. DuBridge, Optical rotation of unpolarized light, J. Opt. Soc. Am. 24, 1 (1934).

[18] R. T. Birge, On the nature of unpolarized light, J. Opt. Soc. Am. 25, 179 (1935).

[19] K. H. Kim, B. H. Park, Y. Tu, T. Hasan, B. Lee, J. Li, and J. F. de Boer, Polarization-sensitive optical frequency domain imaging based on unpolarized light, Opt. Express 19, 552 (2011).

[20] C. Ding, J. R. W. Ulcickas, F. Deng, and G. J. Simpson, Second Harmonic Generation of Unpolarized Light, Phys. Rev. Lett. 119, 193901 (2017).

[21] D. S. Krähmer and U. Leonhardt, Optical homodyne tomography of unpolarized light, Phys. Rev. A 55, 3275 (1997).

[22] T. Richter, Tomographic reconstruction of completely unpolarized light, Phys. Rev. A 55, 4629 (1997).

[23] V. L. Quito and R. Flint, Floquet Engineering Correlated Materials with Unpolarized Light, Phys. Rev. Lett. 126, 177201 (2021).

[24] V. L. Quito and R. Flint, Polarization as a tuning parameter for Floquet engineering: Magnetism in the honeycomb, square, and triangular Mott insulators, Phys. Rev. B 103, 134435 (2021).

[25] G. Piquero, L. Monroy, M. Santarsiero, M. Alonzo, and J. C. G. de Sande, Synthesis of full Poincaré beams by means of uniaxial crystals, J. Opt. 20, 065602 (2018).

[26] G. Piquero, R. Martínez-Herrero, J. C. G. de Sande, and M. Santarsiero, Synthesis and characterization of non-uniformly totally polarized light beams: Tutorial, J. Opt. Soc. Am. A 37, 591 (2020).

[27] A. Luis, Polarization in quantum optics, in Progress in Optics, edited by T. D. Visser, Vol. 61 (Elsevier, Amsterdam, The Netherlands, 2016), Chap. 5, pp. 283-331.

[28] A. Shevchenko, M. Roussey, A. T. Friberg, and T. Setälä, Polarization time of unpolarized light, Optica 4, 64 (2017).

[29] G. G. Kozlov, I. I. Ryzhov, A. Tzimis, Z. Hatzopoulos, P. G. Savvidis, A. V. Kavokin, M. Bayer, and V. S. Zapasskii, Hidden polarization of unpolarized light, Phys. Rev. A 98, 043810 (2018).

[30] A. Hannonen, B. J. Hoenders, W. Elsäßer, A. T. Friberg, and T. Setälä, Ghost polarimetry using Stokes correlations, J. Opt. Soc. Am. A 37, 714 (2020).

[31] M. Rosskopf, T. Mohr, and W. Elsäßer, Ghost Polarization Communication, Phys. Rev. Appl. 13, 034062 (2020).

[32] J. Söderholm, G. Björk, and A. Trifonov, Unpolarized light in quantum optics, Opt. Spectrosc. 91, 532 (2001).

[33] H. Paul and J. Wegmann, Polarization correlations in unpolarized light, Opt. Commun. 112, 85 (1994).

[34] J. Lehner, U. Leonhardt, and H. Paul, Unpolarized light: Classical and quantum states, Phys. Rev. A 53, 2727 (1996).

[35] J. Lehner, H. Paul, and G. Agarwal, Generation and physical properties of a new form of unpolarized light, Opt. Commun. 139, 262 (1997).

[36] A. Luis, Degree of polarization of type-II unpolarized light, Phys. Rev. A 75, 053806 (2007).
[37] J. J. Gil, Polarimetric characterization of light and media, Eur. Phys. J.: Appl. Phys. 40, 1 (2007).

[38] W. Shurcliff and S. Stanley, Polarized Light (Harvard University Press, Cambridge, MA, 1964).

[39] J. Ellis and A. Dogariu, Differentiation of globally unpolarized complex random fields, J. Opt. Soc. Am. A 21, 988 (2004).

[40] M. Eshaghi and A. Dogariu, Discriminating randomly polarized fields, Opt. Lett. 45, 1970 (2020).

[41] F. Kroh, M. Rosskopf, and W. Elsäßer, Ultra-fast Stokes parameter correlations of true unpolarized thermal light: Type-I unpolarized light, Opt. Lett. 45, 5840 (2020).

[42] F. Kroh, M. Rosskopf, and W. Elsäßer, Generation of spatially unpolarized light by a Cornu depolarizer: Equator polarization states as type II unpolarized light, OSA Continuum 4, 1956 (2021).

[43] F. Kroh, M. Rosskopf, and W. Elsäßer, Utilizing a Cornu depolarizer in the generation of spatially unpolarized light, Appl. Opt. 60, 4892 (2021).

[44] C. Brosseau, Fundamentals of Polarized Light: A Statistical Optics Approach (Wiley, New York, 1998).

[45] K. Salazar-Ariza and R. Torres, Trajectories on the Poincaré sphere of polarization states of a beam passing through a rotating linear retarder, J. Opt. Soc. Am. A 35, 65 (2017).

[46] K. Salazar-Ariza and R. Torres, Statistical theory of the polarization on the Poincaré sphere, Opt. Lett. 44, 3318 (2019).

[47] A. Cornu, De la réfraction à travers un prisme suivant une loi quelconque, Annales scientifiques de l'École Normale Supérieure 3, 1 (1874).

[48] A. Cornu, Détermination des trois paramètres optiques principaux d'un cristal, en grandeur et en direction, par le réfractomètre, Bull. Soc. Franç. Minéral. 25, 7 (1902).

[49] M. Griot, Optics Guide (Melles Griot, Inc., Irvine, California, 1988), Vol. 4.

[50] G. G. Stokes, On the composition and resolution of streams of polarized light from different sources, Trans. Cambridge Philos. Soc. 9, 399 (1852), reprinted in Stokes, Mathematical and Physical Papers (Cambridge University Press, Cambridge, 2009).

[51] B. Schaefer, E. Collett, R. Smyth, D. Barrett, and B. Fraher, Measuring the Stokes polarization parameters, Am. J. Phys. 75, 163 (2007).

[52] H. G. Berry, G. Gabrielse, and A. E. Livingston, Measurement of the Stokes parameters of light, Appl. Opt. 16, 3200 (1977).

[53] E. Collett, Polarized Light: Fundamentals and Applications (Marcel Dekker, New York, 1993)

[54] J. P. McGuire and R. A. Chipman, Analysis of spatial pseudodepolarizers in imaging systems, in Polarization Considerations for Optical Systems II, edited by R. A. Chipman (SPIE, Bellingham, WA, 1990), pp. 1478-1484.

[55] We choose here the nomenclature of geodesy, to name the unpolarized circle state on the surface of the Poincaré sphere. In geodetic terms, the "prime meridian" distribution (QWP rotation angle $\alpha=0^{\circ}$ ) is equivalent to the Greenwich meridian. The distributions for QWP rotation angles of $-22.5^{\circ}$ and $-45^{\circ}$ are equivalent to the meridians roughly located at São Paulo (Brazil) and New Orleans (USA), respectively. 